\title{
Borracheras, conducción de vehículos y relaciones sexuales en jóvenes consumidores de cocaína y éxtasis
}

\section{Drunkenness, driving and sexual relations in young cocaine and ecstasy users}

\author{
Elisardo Becoña lgLesias*; Ana López-Durán*; \\ Elena Fernández del Río*; Úrsula Martínez \\ Pradeda*; Jesús Osorio López ${ }^{* *}$; Jaime Fraga \\ Ares*; Manuel Arrojo Romero**; Fernanda \\ López Crecente ${ }^{\star *}$; María Nieves Domínguez \\ GONZÁLEZ $^{\star \star}$
}

Universidad de Santiago de Compostela.

Subdirección General de Gestión Sociosanitaria y Salud Mental, Dirección de Asistencia Sanitaria, Servizo Galego de Saúde, Santiago de Compostela.

Enviar correspondencia a:

Elisardo Becoña Iglesias. Universidad de Santiago de Compostela. Facultad de Psicología. Departamento de Psicología Clínica y

Psicobiología. Campus Universitario Sur.

15782 Santiago de Compostela. A Coruña.

E-mail: elisardo.becona@usc.es

\section{RESUMEN}

Los accidentes de tráfico, las enfermedades de transmisión sexual y embarazos no deseados, las borracheras y el consumo de drogas (fundamentalmente psicoestimulantes) son algunos de los aspectos más negativos asociados al reciente fenómeno de salir de marcha. El objetivo del presente estudio es analizar en una muestra de 1214 jóvenes de 14 a 25 años (49.7\% varones y 50.3\% mujeres) si los consumidores de psicoestimulantes (cocaina y éxtasis) tienen mayor frecuencia de borracheras y de realización de conductas de riesgo en el ámbito de las relaciones sexuales y la conducción de vehículos y analizar el peso que estas conductas tienen para predecir los consumos de psicoestimulantes en el último año. Los resultados indican que los consumidores de psicoestimulantes se emborrachan con más frecuencia, es más probable que hayan visto a un familiar ebrio y tienen más conductas de riesgo relacionadas con la conducción y el mantenimiento de relaciones sexuales completas. Por lo tanto, los consumidores de cocaina y éxtasis son una población de riesgo a la que deben de ir dirigidas campañas de prevención especificas por el alto riesgo de las conductas que realizan.

Palabras clave: cocaina, éxtasis, vida nocturna, accidentes de tráfico, botellón, consumo intensivo de alcohol, relaciones sexuales.
ABSTRACT

Traffic accidents, sexually-transmitted diseases, unwanted pregnancies, drunkenness, and drug use (especially psychostimulants) are negative aspects associated with recreational nightlife. The aim of the present study is to analyze in a sample of 1214 young people (aged 15-25: 49.7\% males, 50.3\% females) whether psychostimulant users (cocaine and ecstasy) have a higher frequency of drunkenness and risk behaviours related to sex and to driving. We also analyze the importance of these behaviours in the prediction of psychostimulant use in the last year. The results indicate that psychostimulant users get drunk more frequently, are more likely to have seen a relative drunk, and present more risk behaviours in the contexts of driving and full sexual relations. Therefore, cocaine and ecstasy users are a risk population who need specific preventive programs.

Key words: cocaine, ecstasy, recreational nightlife, traffic accidents, drunkenness, sexual relationships. 


\section{INTRODUCCIÓN}

L os accidentes de tráfico, las enfermedades de transmisión sexual y embarazos no deseados y el consumo de drogas (Bellis, Hale, Bennet, Chaudry y Kilfoyle, 2000; Hingson, Edwards, Heeren y Rosenbloom, 2009; Miller, Naimi, Brewer y Jones, 2007) son algunas de las consecuencias más sobresalientes del reciente fenómeno de salir de marcha (Calafat et al., 2000) que caracteriza el ocio nocturno de los jóvenes. Estas consecuencias se producen en gran medida por la aparición de nuevos patrones de consumo de sustancias psicoactivas, alcohol, cannabis y otras sustancias como es el caso de los psicoestimulantes (cocaína y éxtasis, fundamentalmente) (Bellis, Hughes, Bennett y Thomson, 2003; Calafat, Fernández, Juan y Becoña, 2005; Calafat et al., 2005; Siliquini, Morra, Versino y Renga, 2005).

En nuestro pais es muy significativo el cambio que se ha producido en las últimas décadas respecto al consumo de alcohol. Tradicionalmente en España era característico el consumo de alcohol de tipo mediterráneo, consistente en pequeños consumos diarios de alcohol. Pero, en la actualidad, el consumo de tipo anglosajón es el que realizan los más jóvenes esto es, grandes cantidades de alcohol en un corto periodo de tiempo (principalmente durante los fines de semana) (Calafat, Fernández, Juan y Becoña, 2005; Cortés, Espejo y Giménez, 2008; Cortés, Espejo, Martín y Gómez, 2010). El consumo excesivo de alcohol es uno de los problemas más importantes que tenemos en la actualidad en la población más joven. Los datos de la encuesta ESTUDES (Observatorio Español sobre Drogas, 2009) del Plan Nacional sobre Drogas, sobre la prevalencia de borracheras en jóvenes españoles de 14 a 18 años, apuntan que el $49.6 \%$ de los estudiantes de secundaria se han emborrachado en alguna ocasión, mientras que en el año 1998 este porcentaje era del $24.2 \%$. La embriaguez es uno de los aspectos clave dentro de los hábitos de salir de marcha en la actualidad. Ha pasado de ser un hecho ocasional y fortuito a ser la meta en sí misma: el joven busca emborracharse, se percibe como un hecho normal y es aceptado con normalidad por los jóvenes (Calafat et al., 2000; Coleman y Cater, 2005). Este tipo de consumo de alcohol tiene importantes consecuencias para la salud de los jóvenes y su desarrollo posterior (Cadaveira, 2009; Steinberg, 2010; Sussman, Skara y Ames, 2008). Además, el consumo se produce dentro del contexto de salir de marcha junto con otras sustancias como son los psicoestimulantes. Cocaina y alcohol se complementan muy bien debido a que sus efectos fisiológicos se potencian, de ahí la alta prevalencia del consumo de alcohol entre los consumidores de cocaina (López y Becoña, 2006a). Además no podemos olvidar que el policonsumo es muy frecuente dentro del contexto de salir de marcha (Observatorio Español sobre Drogas, 2009). Este patrón de consumo está relacionado, además de con las ya conocidas consecuencias negativas para la salud, con otras consecuencias incluso más graves como son los accidentes de tráfico y las conductas sexuales de riesgo (Bellis et al., 2008).

La utilización de vehículos durante la noche para desplazarse a los lugares de marcha es frecuente en nuestro medio. Hacerlo bajo los efectos del alcohol u otro tipo de drogas
(Calafat et al., 2000) puede tener graves consecuencias como son los accidentes de tráfico que con frecuencia tienen un saldo mortal (Farke y Anderson, 2007; Greemblatt, 2000).

El consumo de drogas en el contexto recreativo nocturno también está relacionado con un mayor número de relaciones sexuales (Calafat, Juan, Becoña, Mantecón y Ramón, 2009). Estos consumos son valorados positivamente por parte de los más jóvenes porque consideran estas sustancias como un instrumento facilitador de las relaciones sexuales (Sumnall, Beynon Conchie, Riley y Cole, 2007). En muchos casos el consumo de drogas va asociado a la búsqueda de distintos efectos a la hora de mantener relaciones sexuales: el alcohol es una sustancia valorada positivamente porque facilita el encuentro sexual, involucrarse en experiencias más arriesgadas y aumenta la excitación. La segunda sustancia más valorada por los jóvenes es la cocaína porque permite prolongar la relación sexual (Calafat, Juan, Becoña y Mantecón, 2008).

Pero también encontramos consecuencias negativas derivadas de la asociación entre consumo de drogas y relaciones sexuales. Esto es debido a la disminución de la percepción de riesgo que producen estas sustancias lo que hace más probable mantener relaciones sexuales sin protección, lo cual incrementa los embarazos no deseados y las enfermedades de transmisión sexual (Bellis et al., 2000; Bellis et al., 2008; Coleman y Cater, 2005; Donovan y McEwan, 1995; Rodríguez, Hernán Cabrera, García y Romo, 2007).

En nuestro medio encontramos diversos estudios que analizan las caracteristicas del ocio nocturno en general y las conductas de riesgo asociadas (Bellis e tal., 2008; Calafat, Fernández, Juan y Becoña, 2007; Calafat et al., 2000) y otros centrados en conductas de riesgo concretas como las relaciones sexuales (Becoña et al., 2008; Bellis et al., 2000; Calafat et al., 2008; Calafat et al; 2009; Castilla et al., 1999; Espada et al., 2008).

El objetivo del presente estudio es analizar si los jóvenes consumidores de psicoestimulantes (cocaína y éxtasis), respecto a los que no lo hacen alguna vez en la vida y en el último año, tienen una mayor frecuencia de borracheras y de realización de conductas de riesgo en el ámbito de las relaciones sexuales y la conducción de vehículos. El segundo objetivo es ver el peso que estas conductas tienen para predecir los consumos de psicoestimulantes en el último año.

\section{MÉTODO}

\section{Participantes}

Hemos seleccionado una muestra representativa de jóvenes de las ciudades de Galicia: Santiago de Compostela, Lugo, A Coruña, Ferrol, Pontevedra, Ourense y Vigo. Se ha realizado un muestreo aleatorio, estratificado por las siete ciudades, edad (14-17, 18-21 y 22-25 años), género (hombre, mujer) y consumo-no consumo de psicoestimulantes (cocaína y éxtasis alguna vez en la vida), con entrevistas personales 
en los domicilios de los entrevistados y en los lugares de ocio y diversión para garantizar una adecuada representatividad en función del consumo versus no consumo de psicoestimulantes.

El total de la muestra son 1214 personas. El porcentaje de rechazos para el total de la muestra ha sido del 31.5\%. El número total de entrevistas realizadas en cada ciudad fue el siguiente: 309 en A Coruña (25.4\%), 91 en Ferrol (7.4\%), 117 en Santiago de Compostela (9.6\%), 114 en Lugo (9.3\%), 115 en Ourense (9.4\%), 98 en Pontevedra (8\%) y 370 en Vigo (30.4\%).

De los 1214 sujetos, 603 son varones (49.7\%) y 611 son mujeres (50.3\%). La media de edad de la muestra es de 19.61 años (D. T. $=3.13)$. El 50\% $(n=607)$ ha consumido psicoestimulantes (cocaína y / o éxtasis) alguna vez en la vida [el $33.4 \%(n=406)$ ha consumido éxtasis y el $44.2 \%$ ha consumido cocaína alguna vez $(n=537)]$. Respecto al consumo en el último año, el $37.4 \%$ de la muestra ha consumido psicoestimulantes en el último año [el $22.3 \%(n=271)$ ha consumido éxtasis y el $32.6 \%(n=396)$ consumió cocaína].

\section{Instrumentos de evaluación}

Los jóvenes que participaron en el estudio fueron entrevistados siguiendo un cuestionario estructurado con ítems sobre determinadas conductas relacionadas con salir de marcha. Se evaluó el consumo de psicoestimulantes (cocaína y éxtasis) tanto en el último año como alguna vez en la vida (4 ítems), el consumo elevado de alcohol (frecuencia de borracheras en el último mes y año y ver a algún familiar ebrio) (10 ítems), la conducción de vehículos bajo los efectos del alcohol y otras sustancias (3 items), las posibles consecuencias derivadas de dicha conducta (3 items) y la conducta sexual (edad de inicio de relaciones sexuales completas, número de parejas sexuales en el último año, la realización de conductas sexuales de riesgo y su relación con el consumo de sustancias) (6 ítems). El tiempo medio de aplicación de estos items fue de 15 minutos.

\section{Procedimiento}

Éste es un estudio epidemiológico comunitario transversal en jóvenes gallegos de entre 14 y 25 años. Se realizó siguiendo un muestreo aleatorio en los domicilios de los entrevistados y en lugares de ocio y diversión para garantizar una adecuada representatividad en función del consumo versus no consumo de psicoestimulantes (alguna vez en la vida). Una vez obtenidos los no consumidores (y un adicional porcentaje de consumidores en los domicilios), se completó la muestra en lugares de ocio y diversión buscando en esos lugares a las personas que consumen psicoestimulantes.

En el caso del muestreo en los domicilios, se seleccionaron de forma aleatoria las calles en las que se iba a realizar el estudio en cada una de las ciudades, en función del tamaño de las mismas, y un número de calles de reserva. En el caso de que en alguna de las calles seleccionadas no hubiera viviendas o no habitaran jóvenes de entre 14 y 25 años, la calle era sustituida por una de las de reserva. Se seleccionaba aleatoriamente el primer domicilio habitado para el comienzo del estudio en cada calle. En cada una de las calles el número de entrevistas que se podían hacer era limitado. Los entrevistadores debían de localizar a los jóvenes de 14 a 25 años que vivían en esas calles (deben residir en esa ciudad desde hace seis meses como mínimo), informarles del objeto del estudio y que consintieran participar en el mismo. $Y$ en el caso de los menores de 18 años era preciso localizar a sus padres/tutores para que dieran su consentimiento para que su hijo o tutorizado cumplimentase el cuestionario.

En el caso de los lugares de ocio y diversión, la selección de la muestra fue aleatoria entre los consumidores de estimulantes.

Todas las entrevistas fueron personales y se realizaron por personal especialmente entrenado para ello (psicólogos). En todos los casos, las personas incluidas en el estudio dieron su consentimiento informado. El Comité Ético de Investigación Clínica de la Xunta de Galicia autorizó el presente estudio.

\section{Análisis de datos}

Para el tratamiento estadístico de los datos se ha utilizado el paquete estadístico SPSS para Windows (versión 15.0). Para el análisis de las variables de consumo excesivo de alcohol (borracheras), conducción de vehículos bajo los efectos del alcohol u otras sustancias y conducta sexual en función del consumo de cocaína y éxtasis en el último año y alguna vez en la vida se realizaron tablas de contingencia aplicando el estadístico chi-cuadrado y la t de student en el caso de las variables continuas. El tamaño del efecto se evaluó con la $\mathrm{V}$ de Cramer para la chi-cuadrado y la r para la t de Student (Rosnow y Rosenthal, 1996).

Se realizó también un análisis de regresión logística para comprobar qué variables relacionadas con borracheras, conducción bajo el efecto de sustancias y conducta sexual predicen el consumo de cocaína y éxtasis (en el último año) en jóvenes.

\section{RESULTADOS}

\section{Borracheras en función del consumo de cocaína y éxtasis alguna vez en la vida y en el último año}

Respecto a haberse emborrachado y consumo de cocaína (ver tabla 1), es más probable que los jóvenes que han consumido cocaína alguna vez en la vida se hayan emborrachado en alguna ocasión y lo hayan hecho en el último año y en el último mes un mayor número de veces que los jóvenes que no han consumido nunca cocaína. En cuanto a si han visto a alguien de su familia ebrio, los jóvenes que han consumido cocaína alguna vez en la vida es más probable hayan visto a algún familiar ebrio en comparación a los que nunca consumieron cocaína. Independientemente del parentesco es más 
probable que los consumidores de cocaína hayan visto ebrio a un familiar.

Si comparamos los que han consumido cocaína en el último año con los que no lo han hecho, los resultados van en la misma línea que en el caso del consumo de cocaína alguna vez en la vida (ver Tabla 1).

Respecto a haberse emborrachado y consumo de éxtasis (Tabla 2), es más probable que los jóvenes que han consumido éxtasis alguna vez en la vida se hayan emborrachado en alguna ocasión y lo hayan hecho en el último año y en el último mes un mayor número de veces que los jóvenes que no han consumido nunca éxtasis.

En cuanto a si han visto a alguien de su familia ebrio, los jóvenes que han consumido éxtasis alguna vez en la vida es más probable que hayan visto a algún familiar ebrio en comparación a los que nunca consumieron éxtasis. Independientemente del parentesco es más probable que los consumidores de éxtasis hayan visto ebrio a un familiar.

Tabla 1. Borracheras y consumo de cocaína alguna vez en la vida y en el último año.

\begin{tabular}{|c|c|c|c|c|c|c|c|c|c|c|c|}
\hline \multicolumn{6}{|c|}{ Consumo de cocaína alguna vez en la vida } & \multicolumn{6}{|c|}{ Consumo de cocaína en el último año } \\
\hline \multicolumn{2}{|c|}{ No } & \multicolumn{2}{|c|}{ Si } & & & \multicolumn{2}{|c|}{ No } & \multicolumn{2}{|c|}{$\mathrm{Si}$} & & \\
\hline$n$ & $\%$ & $\mathrm{n}$ & $\%$ & $\chi^{2}$ & V Cramer & $n$ & $\%$ & $n$ & $0 /$ & & V Cramer \\
\hline
\end{tabular}

¿Te has emborrachado alguna vez?

\begin{tabular}{|c|c|c|c|c|c|c|c|c|c|c|c|c|}
\hline No & 157 & 23.3 & 3 & 0.6 & $133.986^{* * *}$ & $0.333^{* * *}$ & 157 & 19.3 & 3 & 0.8 & $79.948^{* * *}$ & $0.257^{* * *}$ \\
\hline \multirow[t]{2}{*}{ Si } & 517 & 76.7 & 531 & 99.4 & & & 655 & 80.7 & 393 & 99.2 & & \\
\hline & Media & D. T. & Media & D. T. & $\mathrm{t}$ & r & Media & D. T. & Media & D. T. & $\mathrm{t}$ & r \\
\hline \multicolumn{13}{|c|}{ ¿Cuántas veces en los últimos 12 meses? } \\
\hline & 21.09 & 29.14 & 44.75 & 50.33 & $-9.292^{* * *}$ & $0.258^{* * *}$ & 20.88 & 29.47 & 50.63 & 540.01 & $-11.821^{* * *}$ & $0.322^{* * *}$ \\
\hline \multicolumn{13}{|c|}{ ¿Yen el último mes? } \\
\hline & 1.68 & 2.38 & 3.46 & 40.01 & $-8.659^{* * *}$ & $0.241^{* * *}$ & 1.67 & 2.40 & 3.87 & 4.33 & $-10.805^{* * *}$ & $0.297^{* * *}$ \\
\hline & $n$ & $\%$ & $n$ & $\%$ & $\chi^{2}$ & V Cramer & $n$ & $\%$ & $n$ & $\%$ & $\chi^{2}$ & V Cramer \\
\hline \multicolumn{13}{|c|}{ ¿Has visto a alguien de tu familia ebrio? } \\
\hline No & 397 & 58.9 & 438 & 82.2 & $75.617^{* * *}$ & $0.250^{* * *}$ & 508 & 62.6 & 327 & 82.8 & $50.972^{* * *}$ & $0.206^{* * *}$ \\
\hline Si & 277 & 41.1 & 95 & 17.8 & & & 304 & 37.4 & 68 & 17.2 & & \\
\hline \multicolumn{13}{|c|}{ ¿Quién era? } \\
\hline \multicolumn{13}{|c|}{ Padre } \\
\hline No & 173 & 27.2 & 257 & 49.4 & $60.772^{* * *}$ & $0.229^{* * *}$ & 224 & 28.9 & 206 & 53.9 & $68.611^{* * *}$ & $0.244^{* * *}$ \\
\hline Si & 464 & 72.8 & 263 & 50.6 & & & 551 & 71.1 & 176 & 46.1 & & \\
\hline \multicolumn{13}{|c|}{ Madre } \\
\hline No & 84 & 13.2 & 151 & 29.1 & $44.683^{* * *}$ & $0.197^{* * *}$ & 116 & 15.0 & 119 & 31.2 & $41.726^{* * *}$ & $0.190^{* * *}$ \\
\hline $\mathrm{Si}$ & 553 & 86.8 & 368 & 70.9 & & & 659 & 85.0 & 262 & 68.8 & & \\
\hline \multicolumn{13}{|c|}{ Hermano/a } \\
\hline No & 190 & 29.8 & 238 & 45.9 & $31.518^{* * *}$ & $0.163^{* * *}$ & 254 & 32.8 & 173 & 45.4 & $17.499^{* * *}$ & $0.123^{* * *}$ \\
\hline Si & 447 & 70.2 & 281 & 54.1 & & & 521 & 67.2 & 208 & 54.6 & & \\
\hline \multicolumn{13}{|c|}{ Tío/a } \\
\hline No & 174 & 27.3 & 225 & 43.4 & $32.542^{* * *}$ & $0.168^{* * *}$ & 226 & 29.2 & 173 & 45.4 & $29.825^{* * *}$ & $0.161^{* * *}$ \\
\hline $\mathrm{Si}$ & 463 & 72.7 & 294 & 56.6 & & & 549 & 70.8 & 208 & 54.6 & & \\
\hline \multicolumn{13}{|c|}{ Abuelo/a } \\
\hline No & 65 & 10.2 & 105 & 20.2 & $22.924^{* * *}$ & $0.141^{* * *}$ & 88 & 11.4 & 82 & 21.5 & $210.052^{* * *}$ & $0.135^{* * *}$ \\
\hline $\mathrm{Si}$ & 572 & 89.8 & 414 & 79.8 & & & 687 & 88.6 & 299 & 78.5 & & \\
\hline \multicolumn{13}{|c|}{ Primo/a } \\
\hline No & 198 & 31.1 & 253 & 48.7 & $37.506^{* * *}$ & $0.180^{* * *}$ & 263 & 33.9 & 188 & 49.3 & $25.488^{* * *}$ & $0.148^{* * *}$ \\
\hline $\mathrm{Si}$ & 439 & 68.9 & 266 & 51.3 & & & 512 & 66.1 & 193 & 50.7 & & \\
\hline \multicolumn{13}{|c|}{ Cuñado/a } \\
\hline No & 64 & 10.0 & 99 & 19.1 & $19.368^{* * *}$ & $0.129^{* * *}$ & 91 & 11.8 & 72 & 18.9 & $10.740^{* *}$ & $0.096^{* * *}$ \\
\hline $\mathrm{Si}$ & 573 & 90.0 & 419 & 80.9 & & & 683 & 88.2 & 309 & 81.1 & & \\
\hline
\end{tabular}

${ }^{*} p<0.05 ;{ }^{* *} p<0.01 ;{ }^{* * *} p<0.001$ 
En el caso de los que han consumido éxtasis el último año respecto a los que no lo han hecho, los resultados van en la misma línea que en el caso del consumo de éxtasis alguna vez en la vida (ver Tabla 2).

\section{Conducción en función del consumo de cocaína y éxtasis alguna vez en la vida y en el último año.}

Respecto a conducción y consumo de cocaína (Tabla 3), es más probable que los jóvenes que han consumido cocaí- na alguna vez en la vida hayan conducido alguna vez en el último año bajo los efectos del alcohol, de alguna otra droga, que hayan ido alguna vez con alguien que conducía bajo los efectos del alcohol y que hayan sido multados, detenidos o que hayan tenido un accidente de tráfico por conducir bajo los efectos del alcohol (él u otra persona), frente a los que nunca han consumido cocaína.

Si comparamos los jóvenes que han consumido cocaina en el último año con los que no lo han hecho encontramos resultados similares (ver Tabla 3 ).

Tabla 2. Borracheras y consumo de éxtasis alguna vez en la vida y en el último año.

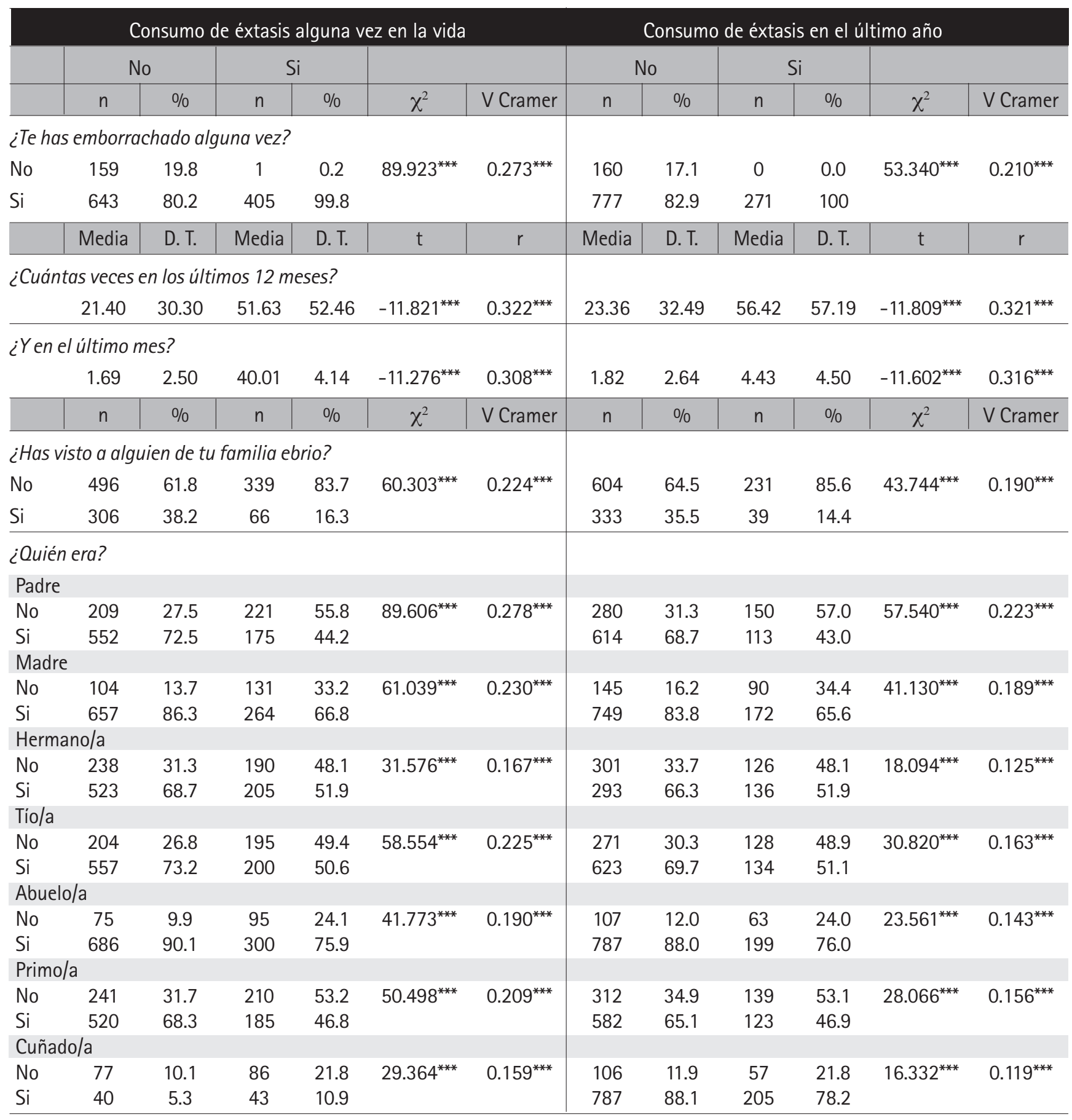

${ }^{*} p<0.05 ;{ }^{* *} p<0.01 ;{ }^{* * *} p<0.001$ 
Tabla 3. Conducción y consumo de cocaína alguna vez en la vida y en el último año.

\begin{tabular}{|c|c|c|c|c|c|c|c|c|c|c|c|}
\hline \multicolumn{6}{|c|}{ Consumo de cocaína alguna vez en la vida } & \multicolumn{6}{|c|}{ Consumo de cocaína en el último año } \\
\hline \multicolumn{2}{|c|}{ No } & \multicolumn{2}{|c|}{ Si } & & & \multicolumn{2}{|c|}{ No } & \multicolumn{2}{|c|}{ Si } & & \\
\hline $\mathrm{n}$ & $\%$ & $n$ & $\%$ & $\chi^{2}$ & V Cramer & $n$ & $\%$ & $\mathrm{n}$ & 0 & & V Cramer \\
\hline
\end{tabular}

En los últimos 12 meses, ¿has conducido alguna vez bajo los efectos del alcohol?

\begin{tabular}{|c|c|c|c|c|c|c|c|c|c|c|c|c|}
\hline No & 619 & 92.3 & 384 & 71.9 & $88.168^{* * *}$ & $0.270^{* * *}$ & 730 & 90.2 & 273 & 68.9 & $86.408^{* * *}$ & $0.268^{* * *}$ \\
\hline $\mathrm{Si}$ & 52 & 7.7 & 150 & 28.1 & & & 79 & 9.8 & 123 & 31.1 & & \\
\hline
\end{tabular}

En los últimos 12 meses, ¿has ido alguna vez con alguien que conducía bajo los efectos del alcohol?

\begin{tabular}{|c|c|c|c|c|c|c|c|c|c|c|c|c|}
\hline No & 478 & 71.2 & 177 & 33.1 & $173.896^{* * *}$ & $0.380^{* * *}$ & 541 & 66.9 & 114 & 28.8 & $155.428^{* * *}$ & $0.359^{* * *}$ \\
\hline $\mathrm{Si}$ & 193 & 28.8 & 357 & 66.9 & & & 268 & 33.1 & 282 & 71.2 & & \\
\hline
\end{tabular}

¿Has tenido alguno de los siguientes problemas a causa de conducir tú u otra persona bajo los efectos del alcohol?

Ser multado

\begin{tabular}{|c|c|c|c|c|c|c|c|c|c|c|c|}
\hline 658 & 98.1 & 453 & 84.1 & $72.377^{* * *}$ & $0.245^{* * *}$ & 784 & 96.9 & 327 & 82.6 & $75.949^{* * *}$ & $0.251^{* * *}$ \\
\hline 13 & 1.9 & 81 & 15.2 & & & 25 & 3.1 & 69 & 17.4 & & \\
\hline \multicolumn{12}{|l|}{ Ser detenido } \\
\hline No $\quad 669$ & 99.7 & 506 & 94.8 & $29.957^{* * *}$ & $0.158^{* * *}$ & 804 & 99.4 & 371 & 93.7 & $35.520^{* * *}$ & $0.172^{* * *}$ \\
\hline 2 & 0.3 & 28 & 5.2 & & & 5 & 0.6 & 25 & 6.3 & & \\
\hline \multicolumn{12}{|c|}{ Accidentes de tráfico } \\
\hline No $\quad 658$ & 98.1 & 474 & 88.8 & $45.176^{* * *}$ & $0.194^{* * *}$ & 785 & 97.0 & 347 & 87.6 & $41.340^{* * *}$ & $0.185^{* * *}$ \\
\hline 13 & 1.9 & 60 & 11.2 & & & 24 & 3.0 & 49 & 12.4 & & \\
\hline \multicolumn{12}{|c|}{ ¿Has conducido alguna vez bajo los efectos de alguna otra droga? } \\
\hline No $\quad 626$ & 93.3 & 335 & 62.7 & $171.961^{* * *}$ & $0.378^{* * *}$ & 729 & 90.1 & 232 & 58.6 & $163.621^{* * *}$ & $0.368^{* * *}$ \\
\hline 45 & 6.7 & 199 & 37.3 & & & 80 & 9.9 & 164 & 41.4 & & \\
\hline
\end{tabular}

${ }^{*} p<0.05 ;{ }^{* *} p<0.01 ;{ }^{* * *} p<0.001$

Tabla 4. Conducción y consumo de éxtasis alguna vez en la vida y en el último año.

\begin{tabular}{|c|c|c|c|c|c|c|c|c|c|c|c|c|}
\hline & \multicolumn{6}{|c|}{ Consumo de éxtasis alguna vez en la vida } & \multicolumn{6}{|c|}{ Consumo de extasis en el último año } \\
\hline & \multicolumn{2}{|c|}{ No } & \multicolumn{2}{|c|}{ Si } & & & \multicolumn{2}{|c|}{ No } & \multicolumn{2}{|c|}{ Si } & \multirow[b]{2}{*}{$\chi^{2}$} & \multirow[b]{2}{*}{ V Cramer } \\
\hline & $n$ & $\%$ & $n$ & $\%$ & $\chi^{2}$ & V Cramer & $n$ & $\%$ & n & $\%$ & & \\
\hline \multicolumn{13}{|c|}{ En los últimos 12 meses, ¿has conducido alguna vez bajo los efectos del alcohol? } \\
\hline No & 722 & 90.4 & 281 & 69.2 & $86.313^{* * *}$ & $0.268^{* * *}$ & 815 & 87.3 & 188 & 69.4 & $48.161^{* * *}$ & $0.200^{* * *}$ \\
\hline $\mathrm{Si}$ & 77 & 9.6 & 125 & 30.8 & & & 119 & 12.7 & 83 & 30.6 & & \\
\hline
\end{tabular}

En los últimos 12 meses, ¿has ido alguna vez con alguien que conducía bajo los efectos del alcohol?

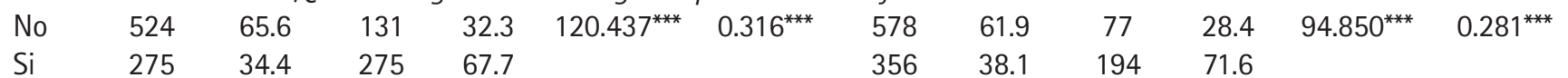

¿Has tenido alguno de los siguientes problemas a causa de conducir tú u otra persona bajo los efectos del alcohol?

\section{Ser multado}

\begin{tabular}{|c|c|c|c|c|c|c|c|c|c|c|c|c|}
\hline No & 771 & 96.5 & 340 & 83.7 & $60.864^{* * *}$ & $0.225^{* * *}$ & 885 & 94.8 & 226 & 83.4 & $37.682^{* * *}$ & $0.177^{* * *}$ \\
\hline Si & 28 & 3.5 & 66 & 16.3 & & & 49 & 5.2 & 45 & 16.6 & & \\
\hline \multicolumn{13}{|c|}{ Ser detenido } \\
\hline No & 793 & 99.2 & 382 & 94.1 & $29.530^{* * *}$ & $0.157^{* * *}$ & 922 & 98.7 & 253 & 93.4 & $24.833^{* * *}$ & $0.144^{* * *}$ \\
\hline Si & 6 & 0.8 & 24 & 5.9 & & & 12 & 1.3 & 18 & 6.6 & & \\
\hline \multicolumn{13}{|c|}{ Accidentes de tráfico } \\
\hline No & 777 & 97.2 & 355 & 87.4 & $45.505^{* * *}$ & $0.194^{* * *}$ & 900 & 96.4 & 232 & 85.6 & $42.660^{* * *}$ & $0.188^{* * *}$ \\
\hline Sí & 22 & 2.8 & 51 & 12.6 & & & 34 & 3.6 & 39 & 14.4 & & \\
\hline \multicolumn{13}{|c|}{ ¿Has conducido alguna vez bajo los efectos de alguna otra droga? } \\
\hline No & 727 & 91.0 & 234 & 57.6 & $185.449^{* * *}$ & $0.392^{* * *}$ & 805 & 86.2 & 156 & 57.6 & $106.573^{* * *}$ & $0.297^{* * *}$ \\
\hline Sí & 72 & 9.0 & 172 & 42.4 & & & 129 & 13.8 & 115 & 42.4 & & \\
\hline
\end{tabular}

${ }^{*} p<0.05 ;{ }^{* *} p<0.01 ; * * * 0.001$ 
Respecto a conducción y consumo de éxtasis (Tabla 4), es más probable que los jóvenes que han consumido éxtasis alguna vez en la vida hayan conducido alguna vez en el último año bajo los efectos del alcohol, de alguna otra droga, que hayan ido alguna vez con alguien que conducía bajo los efectos del alcohol y que hayan sido multados, detenidos o que hayan tenido un accidente de tráfico por conducir bajo los efectos del alcohol (él u otra persona), frente a los que nunca han consumido éxtasis.

Si comparamos los jóvenes que han consumido éxtasis en el último año con los que no lo han hecho encontramos resultados similares (ver Tabla 4).

Relaciones sexuales en función del consumo de cocaína y éxtasis alguna vez en la vida y en el último año.
En cuanto a si han tenido relaciones sexuales completas (Tabla 5), es más probable que los que han consumido cocaína alguna vez en la vida hayan tenido relaciones sexuales completas, que mantuvieran la primera relación sexual a menor edad, que en el último año hayan mantenido relaciones sexuales con un mayor número de personas, es más probable que no utilicen preservativo ni otra forma de control del embarazo y que hayan mantenido relaciones sexuales bajo los efectos del alcohol y de otras drogas.

En la comparación entre consumidores de cocaína en el último año y no consumidores los resultados van en la misma línea de los vistos para alguna vez en la vida (ver Tabla 5).

En cuanto a los jóvenes que han consumido éxtasis alguna vez en la vida (Tabla 6), es más probable que hayan tenido relaciones sexuales completas, que mantuvieran la primera

Tabla 5. Relaciones sexuales y consumo de cocaína alguna vez en la vida y en el último año.

\begin{tabular}{|c|c|c|c|c|c|c|c|c|c|c|c|c|}
\hline & \multicolumn{6}{|c|}{ Consumo de cocaína alguna vez en la vida } & \multicolumn{6}{|c|}{ Consumo de cocaina en el último año } \\
\hline & \multicolumn{2}{|c|}{ No } & \multicolumn{2}{|c|}{ Si } & \multirow[b]{2}{*}{$\chi^{2}$} & \multirow[b]{2}{*}{ V Cramer } & \multicolumn{2}{|c|}{ No } & \multicolumn{2}{|c|}{ Si } & \multirow[b]{2}{*}{$\chi^{2}$} & \multirow[b]{2}{*}{ V Cramer } \\
\hline & $n$ & $\%$ & $n$ & $\%$ & & & $n$ & $\%$ & $n$ & $\%$ & & \\
\hline \multicolumn{13}{|c|}{ ¿Has tenido alguna vez relaciones sexuales completas? } \\
\hline No & 180 & 26.6 & 22 & 4.1 & $109.212^{* * *}$ & $0.300^{* * *}$ & 184 & 22.5 & 18 & 4.5 & $61.971^{* * *}$ & $0.226^{* * *}$ \\
\hline \multirow[t]{2}{*}{ Si } & 497 & 73.4 & 515 & 95.9 & & & 634 & 77.5 & 378 & 95.5 & & \\
\hline & Media & D. T. & Media & D. T. & $\mathrm{t}$ & r & Media & D. T. & Media & D. T. & $t$ & r \\
\hline \multicolumn{13}{|c|}{ ¿Qué edad tenías? } \\
\hline & 16.40 & 1.66 & 15.49 & 1.87 & $8.153^{* * *}$ & $0.248^{* * *}$ & 16.37 & 1.72 & 15.23 & 1.78 & $10.028^{* * *}$ & $0.300^{* * *}$ \\
\hline
\end{tabular}

¿Con cuántas personas distintas has tenido relaciones sexuales en el último año?

$\begin{array}{llllllllllll}2.22 & 2.48 & 3.71 & 4.59 & -6.213^{* * *} & 0.191^{* * *} & 2.09 & 2.40 & 4.10 & 50.05 & -8.513^{* * *} & 0.258^{* * *}\end{array}$

\begin{tabular}{l|l|l|l|l|l|l|l|l|l|l|l|l}
$\mathrm{n}$ & $\%$ & $\mathrm{n}$ & $\%$ & $\chi^{2}$ & V Cramer & $\mathrm{n}$ & $\%$ & $\mathrm{n}$ & $\%$ & $\chi^{2}$ & V Cramer \\
\hline
\end{tabular}

¿Con qué frecuencia has tenido relaciones sexuales sin usar preservativo?

\begin{tabular}{|c|c|c|c|c|c|c|c|c|c|c|c|c|}
\hline Nunca & 233 & 49.3 & 143 & 28.4 & $49.213^{* * *}$ & $0.223^{* * *}$ & 285 & 47.0 & 92 & 24.9 & $50.886^{* * *}$ & $0.228^{* * *}$ \\
\hline Alg. vez & 136 & 28.8 & 172 & 34.2 & & & 171 & 28.2 & 137 & 37.0 & & \\
\hline La may. & 47 & 9.9 & 80 & 15.9 & & & 59 & 9.7 & 68 & 18.4 & & \\
\hline Siempre & 57 & 12.1 & 108 & 21.5 & & & 92 & 15.2 & 73 & 19.7 & & \\
\hline
\end{tabular}

¿Con qué frecuencia has tenido relaciones sexuales sin utilizar alguna forma de control del embarazo?

\begin{tabular}{|c|c|c|c|c|c|c|c|c|c|c|c|c|}
\hline Nunca & 334 & 70.6 & 269 & 53.5 & $41.071^{* * *}$ & $0.206^{* * *}$ & 421 & 69.4 & 182 & 49.2 & $50.287^{* * *}$ & $0.227^{* * *}$ \\
\hline Alg. vez & 107 & 22.6 & 142 & 28.2 & & & 136 & 22.4 & 114 & 30.8 & & \\
\hline La may. & 14 & 3.0 & 51 & 10.1 & & & 21 & 3.5 & 44 & 11.9 & & \\
\hline Siempre & 18 & 3.8 & 41 & 8.2 & & & 29 & 4.8 & 30 & 8.1 & & \\
\hline
\end{tabular}

¿Con qué frecuencia has tenido relaciones sexuales bajo el efecto del alcohol?

\begin{tabular}{|c|c|c|c|c|c|c|c|c|c|c|c|c|}
\hline Nunca & 176 & 37.2 & 78 & 15.6 & $80.168^{* * *}$ & $0.288^{* * *}$ & 203 & 33.4 & 51 & 13.9 & $83.878^{* * *}$ & $0.293^{* * *}$ \\
\hline Alg. vez & 258 & 54.5 & 306 & 61.1 & & & 349 & 57.5 & 215 & 58.4 & & \\
\hline La may. & 31 & 6.6 & 94 & 18.8 & & & 46 & 7.6 & 80 & 21.7 & & \\
\hline Siempre & 8 & 1.7 & 23 & 4.6 & & & 9 & 1.5 & 22 & 6.0 & & \\
\hline
\end{tabular}

¿Con qué frecuencia has tenido relaciones sexuales bajo el efecto de drogas?

\begin{tabular}{|c|c|c|c|c|c|c|c|c|c|c|c|c|}
\hline Nunca & 380 & 80.3 & 200 & 39.8 & $168.513^{* * *}$ & $0.416^{* * *}$ & 465 & 76.6 & 115 & 31.1 & $198.516^{* * *}$ & $0.451^{* * *}$ \\
\hline Alg. vez & 73 & 15.4 & 216 & 42.9 & & & 104 & 17.1 & 185 & 50.0 & & \\
\hline La may. & 17 & 3.6 & 64 & 12.7 & & & 31 & 5.1 & 51 & 13.8 & & \\
\hline Siempre & 3 & 0.6 & 23 & 4.6 & & & 7 & 1.2 & 19 & 5.1 & & \\
\hline
\end{tabular}

${ }^{*} p<0.05 ;{ }^{* *} p<0.01 ; * * * 0.001$ 
Tabla 6. Relaciones sexuales y consumo de éxtasis alguna vez en la vida y en el último año.

\begin{tabular}{|c|c|c|c|c|c|c|c|c|c|c|c|c|}
\hline & & nsumo & éxtasis & lguna v & $z$ en la vida & & & onsum & de éxtas & en el $i$ & imo año & \\
\hline & & & S & & & & & & & & & \\
\hline & $n$ & $\%$ & $n$ & $\%$ & $\chi^{2}$ & V Cramer & $n$ & $\%$ & $n$ & $\%$ & $\chi^{2}$ & V Cramer \\
\hline ¿Has ten & nido alg & $a$ vez $r$ & ycioness & xuales & mpletas? & & & & & & & \\
\hline No & 185 & 22.9 & 17 & 4.2 & $68.190^{* * *}$ & $0.237^{* * *}$ & 190 & 20.1 & 12 & 4.4 & $37.506^{* * *}$ & $0.176^{* * *}$ \\
\hline $\mathrm{Si}$ & 623 & 77.1 & 389 & 95.8 & & & 753 & 79.9 & 259 & 95.6 & & \\
\hline & Media & D. T. & Media & D. T. & $\mathrm{t}$ & r & Media & D. T. & Media & D. T. & $t$ & $r$ \\
\hline ¿Qué ed & ad tenic & & & & & & & & & & & \\
\hline & 16.34 & 1.75 & 15.30 & 1.77 & $9.099^{* * *}$ & $0.275^{* * *}$ & 16.25 & 1.77 & 15.03 & 1.69 & $9.710^{* * *}$ & $0.292^{* * *}$ \\
\hline ¿Con cuc & ántas p & onaso & intas ha & tenido & laciones se & yales en el & Itimo añ & & & & & \\
\hline & 2.25 & 2.46 & 4.13 & 50.01 & $-7.716^{* * *}$ & $0.235^{* * *}$ & 2.23 & 2.51 & 4.62 & 5.68 & $-9.192^{* * *}$ & $0.277^{* * *}$ \\
\hline & $n$ & $\%$ & $n$ & $\%$ & $\chi^{2}$ & V Cramer & $n$ & $\%$ & $n$ & $\%$ & $\chi^{2}$ & V Cramer \\
\hline ¿Con que & é frecue & ia has & ido relo & iones $\mathrm{s}$ & uales $\sin u$ & or preservat & & & & & & \\
\hline Nunca & 278 & 46.6 & 98 & 25.8 & $51.981^{* * *}$ & $0.231^{* * *}$ & 309 & 42.7 & 68 & 26.9 & $29.276^{* * *}$ & $0.173^{* * *}$ \\
\hline Alg. vez & 176 & 29.5 & 132 & 34.7 & & & 217 & 30.0 & 91 & 36.0 & & \\
\hline La may. & 53 & 8.9 & 4 & 19.5 & & & 75 & 10.4 & 52 & 20.6 & & \\
\hline Siempre & 89 & 14.9 & 76 & 20.0 & & & 123 & 17.0 & 42 & 16.6 & & \\
\hline
\end{tabular}

¿Con qué frecuencia has tenido relaciones sexuales sin utilizar alguna forma de control del embarazo?

\begin{tabular}{|c|c|c|c|c|c|c|c|c|c|c|c|c|}
\hline Nunca & 417 & 70.0 & 186 & 48.9 & $50.009^{* * *}$ & $0.226^{* * *}$ & 483 & 66.7 & 120 & 47.4 & $32.866^{* * *}$ & $0.183^{* * *}$ \\
\hline Alg. vez & 130 & 21.8 & 119 & 31.3 & & & 166 & 22.9 & 84 & 33.2 & & \\
\hline La may. & 23 & 3.9 & 42 & 11.1 & & & 36 & 5.0 & 29 & 11.5 & & \\
\hline Siempre & 26 & 4.4 & 33 & 8.7 & & & 39 & 5.4 & 20 & 7.9 & & \\
\hline
\end{tabular}

¿Con qué frecuencia has tenido relaciones sexuales bajo el efecto del alcohol?

\begin{tabular}{|c|c|c|c|c|c|c|c|c|c|c|c|c|}
\hline Nunca & 200 & 33.6 & 54 & 14.2 & $79.654^{* * *}$ & $0.284^{* * *}$ & 216 & 29.9 & 38 & 15.0 & $60.195^{* * *}$ & $0.248^{* * *}$ \\
\hline Alg. vez & 341 & 57.3 & 223 & 58.8 & & & 426 & 59.0 & 138 & 54.5 & & \\
\hline La may. & 42 & 7.1 & 83 & 21.9 & & & 64 & 8.9 & 62 & 24.5 & & \\
\hline Siempre & 12 & 2.0 & 19 & 5.0 & & & 16 & 2.2 & 15 & 5.9 & & \\
\hline
\end{tabular}

¿Con qué frecuencia has tenido relaciones sexuales bajo el efecto de drogas?

\begin{tabular}{|c|c|c|c|c|c|c|c|c|c|c|c|c|}
\hline Nunca & 459 & 77.0 & 121 & 31.8 & $200.595^{* * *}$ & $0.451^{* * *}$ & 514 & 71.0 & 66 & 26.1 & $162.423^{* * *}$ & $0.408^{* * *}$ \\
\hline Alg. vez & 107 & 18.0 & 182 & 47.9 & & & 160 & 22.1 & 129 & 51.0 & & \\
\hline La may.a & 20 & 3.4 & 61 & 16.1 & & & 35 & 4.8 & 47 & 18.6 & & \\
\hline Siempre & 10 & 1.7 & 16 & 4.2 & & & 15 & 2.1 & 11 & 4.3 & & \\
\hline
\end{tabular}

${ }^{*} p<0.05 ;{ }^{* *} p<0.01 ;{ }^{* *} p<0.001$

relación sexual a menor edad, que en el último año hayan mantenido relaciones sexuales con un mayor número de personas, es más probable que no utilicen preservativo ni otra forma de control del embarazo y que hayan mantenido relaciones sexuales bajo los efectos del alcohol y de otras drogas, frente a los que nunca han consumido éxtasis.

En la comparación entre consumidores de éxtasis en el último año y no consumidores los resultados van en la misma línea de los vistos para alguna vez en la vida (ver Tabla 6).

\section{Análisis de regresión logística}

Hemos realizado un análisis de regresión logística introduciendo como variables predictoras las variables relacionadas con borracheras, conducción y relaciones sexuales. Hemos establecido dos grupos de variables predictoras: en el primer grupo (A) se incluyen las variables relacionadas con las borracheras, la conducción y únicamente el haber mantenido o no relaciones sexuales completas $(n=1069)$ (Variables predictoras: ¿Te has emborrachado alguna vez?; ¿Cuántas veces en los últimos 12 meses?; ¿Y en el último mes?; ¿Has visto a alguien de tu familia ebrio?; ¿Quién era? Padre, Madre, Hermano/a, Tío/a, Abuelo/a, Primo/a, Cuñado/a, Hermano/a; En los últimos 12 meses, ¿has conducido alguna vez bajo los efectos del alcohol?; En los últimos 12 meses, ¿has ido alguna vez con alguien que conducía bajo los efectos del alcohol?; ¿Has tenido alguno de los siguientes problemas a causa de conducir tú u otra persona bajo los efectos del alcohol? Ser multado, Ser detenido, Accidentes de tráfico; ¿Has conducido alguna vez bajo los efectos de alguna otra droga?; ¿Has tenido alguna vez relaciones sexuales completas?). 
Tabla 7. Regresión logística del consumo de cocaína en el último año.

\begin{tabular}{|c|c|c|c|c|c|c|c|}
\hline \multirow[b]{2}{*}{ Regresión logística A } & \multicolumn{7}{|c|}{ Variables en la ecuación } \\
\hline & B & E.T. & Wald & gl & Sig. & $\operatorname{Exp}(B)$ & I.C. (95\%) \\
\hline ¿Te has emborrachado alguna vez? & 2.391 & 1.017 & 5.523 & 1 & 0.019 & 10.922 & $(1.487-8.203)$ \\
\hline $\begin{array}{l}\text { ¿Cuántas veces en los últimos } 12 \text { meses } \\
\text { te has emborrachado? }\end{array}$ & 0.013 & 0.002 & 32.497 & 1 & 0.001 & 1.013 & $(1.008-1.017)$ \\
\hline Ver ebrio al padre & 0.466 & 0.152 & 9.358 & 1 & 0.002 & 1.593 & $(1.182-2.147)$ \\
\hline $\begin{array}{l}\text { En los últimos } 12 \text { meses, ¿has ido alguna vez } \\
\text { con alguien que conducía bajo los efectos } \\
\text { del alcohol? }\end{array}$ & 0.723 & 0.161 & 22.267 & 1 & 0.001 & 2.061 & $(1.504-2.823)$ \\
\hline $\begin{array}{l}\text { ¿Has tenido alguno de los siguientes problemas } \\
\text { a causa de conducir tú u otra persona bajo los } \\
\text { efectos del alcohol? - Ser multado }\end{array}$ & 0.710 & 0.282 & 6.319 & 1 & 0.012 & 2.033 & $(1.169-3.537)$ \\
\hline $\begin{array}{l}\text { ¿Has tenido alguno de los siguientes problemas } \\
\text { a causa de conducir tú u otra persona bajo los } \\
\text { efectos del alcohol? - Ser detenido }\end{array}$ & 1.298 & 0.592 & 4.809 & 1 & 0.028 & 3.661 & $(1.148-11.678)$ \\
\hline $\begin{array}{l}\text { ¿Has conducido alguna vez bajo los efectos } \\
\text { de alguna otra droga? }\end{array}$ & 1.078 & 0.177 & 37.020 & 1 & 0.001 & 2.937 & $(2.076-4.156)$ \\
\hline Constante & -4.312 & 1.013 & 18.129 & 1 & 0.001 & 0.013 & \\
\hline \multicolumn{8}{|l|}{ Regresión logística B } \\
\hline $\begin{array}{l}\text { ¿Cuántas veces en los últimos } 12 \text { meses } \\
\text { te has emborrachado? }\end{array}$ & 0.010 & 0.002 & 19.431 & 1 & 0.001 & 1.010 & $(1.006-1.015)$ \\
\hline $\begin{array}{l}\text { En los últimos } 12 \text { meses, ihas ido alguna vez } \\
\text { con alguien que conducia bajo los efectos } \\
\text { del alcohol? }\end{array}$ & 0.564 & 0.179 & 9.960 & 1 & 0.002 & 1.758 & $(1.238-2.495)$ \\
\hline $\begin{array}{l}\text { ¿Has tenido alguno de los siguientes problemas } \\
\text { a causa de conducir tú u otra persona bajo los } \\
\text { efectos del alcohol? - Ser multado }\end{array}$ & 0.723 & 0.292 & 6.111 & 1 & 0.013 & 2.061 & $(1.162-3.656)$ \\
\hline $\begin{array}{l}\text { ¿Has conducido alguna vez bajo los efectos } \\
\text { de alguna otra droga? }\end{array}$ & 0.730 & 0.197 & 13.695 & 1 & 0.001 & 2.075 & (1.410-3.054) \\
\hline $\begin{array}{l}\text { ¿Qué edad tenías cuando empezaste a mantener } \\
\text { relaciones sexuales completas? }\end{array}$ & -0.257 & 00.050 & 26.161 & 1 & 0.001 & 0.773 & $(0.701-0.853)$ \\
\hline $\begin{array}{l}\text { ¿Con cuántas personas distintas has tenido } \\
\text { relaciones sexuales en el último año? }\end{array}$ & 0.057 & 0.026 & 4.722 & 1 & 0.030 & 1.059 & $(1.006-1.115)$ \\
\hline $\begin{array}{l}\text { ¿Con qué frecuencia has tenido relaciones } \\
\text { sexuales sin usar preservativo? }\end{array}$ & 0.198 & 0.076 & 6.758 & 1 & 0.009 & 1.219 & $(1.050-1.416)$ \\
\hline $\begin{array}{l}\text { ¿Con qué frecuencia has tenido relaciones } \\
\text { sexuales bajo el efecto de drogas? }\end{array}$ & 0.564 & 0.121 & 21.738 & 1 & 0.001 & 1.758 & $(1.387-2.229)$ \\
\hline Constante & 1.130 & 0.859 & 1.729 & 1 & 0.189 & 3.095 & \\
\hline
\end{tabular}

En el segundo grupo (B) se han introducido todas las variables relacionadas con las relaciones sexuales $(n=915)$ (Variables predictoras: ¿Te has emborrachado alguna vez?; ¿Cuántas veces en los últimos 12 meses?; ¿Y en el último mes?; ¿Has visto a alguien de tu familia ebrio?; ¿Quién era? Padre, Madre, Hermano/a, Tío/a, Abuelo/a, Primo/a, Cuñado/a, Hermano/a; En los últimos 12 meses, ¿has conducido alguna vez bajo los efectos del alcohol?; En los últimos
12 meses, ¿has ido alguna vez con alguien que conducía bajo los efectos del alcohol?; ¿Has tenido alguno de los siguientes problemas a causa de conducir tú u otra persona bajo los efectos del alcohol? Ser multado, Ser detenido, Accidentes de tráfico; ¿Has conducido alguna vez bajo los efectos de alguna otra droga?; ¿ ¿Has tenido alguna vez relaciones sexuales completas?; ¿Qué edad tenías?; ¿Con cuántas personas distintas has tenido relaciones sexuales en el último año?; ¿Con 
qué frecuencia has tenido relaciones sexuales sin usar preservativo?; ¿Con qué frecuencia has tenido relaciones sexuales sin utilizar alguna forma de control del embarazo?; ¿Con qué frecuencia has tenido relaciones sexuales bajo el efecto del alcohol?; ¿Con qué frecuencia has tenido relaciones sexuales bajo el efecto de drogas?). En este caso el tamaño de la muestra es menor porque todos aquellos jóvenes que no han mantenido relaciones sexuales completas son eliminados del análisis de regresión porque no pudieron responder al resto de las preguntas relacionadas con la conducta sexual. Con estas variables haremos los análisis para consumo de cocaína (Tabla 7) y consumo de éxtasis (Tabla 8) en el último año.

\section{a) Análisis de regresión logística entre borracheras, conducción, relaciones sexuales y consumo de cocaína en el último año}

Utilizando como variable criterio el consumo - no consumo de cocaína en el último año encontramos, en el análisis de regresión A (ver Tabla 7), que haberse emborrachado alguna vez, haberse emborrachado un mayor número de veces en el último año, haber visto a su padre ebrio, haber ido en el último año con alguien que conducía bajo los efectos del alcohol, haber sido multado o haber sido detenido por conducir bajo los efectos del alcohol o ir con alguien que estaba bajo los efectos de esa sustancia y haber conducido alguna vez bajo los efectos de otro tipo de sustancias psicoactivas incrementa la probabilidad de haber consumido cocaína en el último año. Se clasifican correctamente el $73.6 \%$ de los jóvenes evaluados.

En el análisis de regresión B (ver Tabla 7) añadimos la totalidad de las variables referidas a la conducta sexual. Encontramos que haberse emborrachado un mayor número de veces en el último año, haber ido en el último año con alguien que conducia bajo los efectos del alcohol, haber sido multado por conducir bajo los efectos del alcohol, o ir con alguien que estaba bajo los efectos de esa sustancia, haber conducido alguna vez bajo los efectos de otro tipo de sustancias psicoactivas, mantener relaciones sexuales completas a una edad más temprana, haber tenido relaciones sexuales con un mayor número de parejas durante el último año, tener una mayor frecuencia de relaciones sexuales sin utilizar preservativo y una mayor frecuencia de relaciones sexuales bajo el efecto de drogas incrementa la probabilidad de haber consumido cocaína en el último año. Se clasifican correctamente el $75.5 \%$ de los jóvenes evaluados.

\section{b) Análisis de regresión logística entre borracheras, conducción y relaciones sexuales y consumo de éxtasis en el último año}

Utilizando como variable criterio el consumo - no consumo de éxtasis en el último año encontramos, en el análisis de regresión A (ver Tabla 8), que haberse emborrachado un mayor número de veces en el último año $(O R=1.008)$ y en el último mes $(O R=1.110)$, haber visto a su padre ebrio (OR = 1.668), haber ido en el último año con alguien que conducía bajo los efectos del alcohol $(O R=1.664)$, haber tenido un accidente de tráfico por haber ido bajo los efectos del alcohol.(OR $=1.739)$ y haber conducido alguna vez bajo los efectos de otro tipo de sustancias psicoactivas (OR $=2.324$ ) incrementa la probabilidad de haber consumido éxtasis en el último año. Se clasifican correctamente el $77.4 \%$ de los jóvenes evaluados.

En el análisis de regresión B (ver Tabla 8) encontramos que haberse emborrachado un mayor número de veces en el último año $(O R=1.011)$, haber conducido alguna vez bajo los efectos de otro tipo de sustancias psicoactivas distintas al alcohol $(O R=1.625)$, haber visto ebrio a su padre $(O R=$ 1.422), mantener relaciones sexuales completas a una edad más temprana $(O R=0.743)$, haber tenido relaciones sexuales con un mayor número de parejas durante el último año (OR $=1.074)$ y una mayor frecuencia de relaciones sexuales bajo el efecto de drogas $(O R=1.841)$ incrementa la probabilidad de haber consumido éxtasis en el último año. Se clasifican correctamente el 78.6\% de los jóvenes evaluados.

\section{DISCUSIÓN}

En el presente estudio encontramos que existe relación entre el consumo de psicoestimulantes (cocaína y éxtasis) y determinadas conductas con efectos negativos para la salud, como son el consumo intenso de alcohol (borracheras), los accidentes de tráfico y las conductas sexuales de riesgo.

Respecto al consumo de alcohol, es más probable que los jóvenes que han consumido cocaína o éxtasis se hayan emborrachado alguna vez y que en el último año y mes lo hayan hecho con una mayor frecuencia. Esto indica una clara asociación entre borracheras y consumo de psicoestimulantes entre los jóvenes. Como señalábamos previamente, el policonsumo es frecuente en el contexto de salir de marcha (Observatorio Español sobre Drogas, 2009) y concretamente el alcohol y la cocaína son dos sustancias que se complementan a la perfección (López y Becoña, 2006b).

El consumo de alcohol es un problema relevante entre los jóvenes en la actualidad. En el estudio de Ballester y Gil (2009) el 60\% de los jóvenes entrevistados señalaban que consumian cantidades elevadas de alcohol habitualmente durante los fines de semana, siendo las principales motivaciones para su consumo: facilitar la diversión, las relaciones sociales y evadirse de los problemas. Hughes, Bellis, Whelan, Calafat, Juan y Blay (2009) y Schnitzer et al. (2010) señalan que hay una estrecha relación entre el consumo de altas cantidades de alcohol y conductas violentas (peleas, discusiones) e incluso la necesidad de recibir atención médica. Hingson et al. (2009) y Miller et al. (2007) coinciden en señalar que emborracharse está relacionado con diversas conductas negativas para la salud como ir en un coche que conduce alguien que ha bebido alcohol, mantener relaciones sexuales de riesgo, accidentes de tráfico, peleas, fumar y consumir drogas. 
Tabla 8. Regresión logística del consumo de éxtasis en el último año.

\begin{tabular}{|c|c|c|c|c|c|c|c|}
\hline \multirow[b]{2}{*}{ Regresión logística A } & \multicolumn{7}{|c|}{ Variables en la ecuación } \\
\hline & B & E.T. & Wald & gl & Sig. & $\operatorname{Exp}(B)$ & I.C. $(95 \%)$ \\
\hline ¿Cuántas veces en los últimos 12 meses? & 0.008 & 0.003 & 6.475 & 1 & 0.011 & 1.008 & $(1.002-1.014)$ \\
\hline ¿Y en el último mes? & 0.105 & 0.040 & 6.926 & 1 & 0.008 & 1.110 & $(1.027-1.200)$ \\
\hline Ver ebrio al padre & 0.512 & 0.163 & 9.882 & 1 & 0.002 & 1.668 & $(1.213-2.296)$ \\
\hline $\begin{array}{l}\text { En los últimos } 12 \text { meses, ¿has ido alguna } \\
\text { vez con alguien que conducía bajo los efectos } \\
\text { del alcohol? }\end{array}$ & 0.509 & 0.179 & 8.141 & 1 & 0.004 & 1.664 & $(1.173-2.361)$ \\
\hline $\begin{array}{l}\text { ¿Has tenido alguno de los siguientes problemas } \\
\text { a causa de conducir tú u otra persona bajo los } \\
\text { efectos del alcohol? Accidentes de tráfico. }\end{array}$ & 0.553 & 0.282 & 3.858 & 1 & 0.049 & 1.739 & $(1.001-3.020)$ \\
\hline $\begin{array}{l}\text { ¿Has conducido alguna vez bajo los efectos de } \\
\text { alguna otra droga? }\end{array}$ & 0.843 & 0.179 & 22.257 & 1 & 0.000 & 2.324 & $(1.637-3.298)$ \\
\hline Constante & -2.5138 & 0.157 & 256.911 & 1 & 0.000 & 0.081 & \\
\hline \multicolumn{8}{|l|}{ Regresión logística B } \\
\hline ¿Cuántas veces en los últimos 12 meses? & 0.011 & 0.002 & 26.065 & 1 & 0.001 & 1.011 & $(1.007-1.016)$ \\
\hline Ver ebrio al padre & 0.352 & 0.179 & 3.878 & 1 & 0.049 & 1.422 & $(1.002-2.018)$ \\
\hline $\begin{array}{l}\text { ¿Has conducido alguna vez bajo los efectos } \\
\text { de alguna otra droga? }\end{array}$ & 0.486 & 0.197 & 6.104 & 1 & 00.13 & 1.625 & $(1.106-2.389)$ \\
\hline $\begin{array}{l}\text { ¿Qué edad tenías cuando empezaste a } \\
\text { mantener relaciones sexuales completas? }\end{array}$ & -0.297 & 00.054 & 30.635 & 1 & 0.001 & 0.743 & $(0.669-0.826)$ \\
\hline $\begin{array}{l}\text { ¿Con cuántas personas distintas has tenido } \\
\text { relaciones sexuales en el último año? }\end{array}$ & 0.071 & 0.024 & 9.104 & 1 & 0.003 & 1.074 & $(1.025-1.125)$ \\
\hline $\begin{array}{l}\text { ¿Con qué frecuencia has tenido relaciones } \\
\text { sexuales bajo el efecto de drogas? }\end{array}$ & 0.610 & 0.117 & 27.004 & 1 & 0.001 & 1.841 & $(1.463-2.318)$ \\
\hline Constante & 1.623 & 0.879 & 3.407 & 1 & 0.065 & 5.068 & \\
\hline
\end{tabular}

Respecto a si han visto a algún familiar ebrio, también es más probable que los que han consumido psicoestimulantes hayan visto borracho a algún familiar. Y son los padres y los primos, a los que han visto borrachos con mayor frecuencia. Estos resultados subrayan la importancia que tiene la familia como modelo para el futuro consumo de alcohol en los jóvenes (Velleman, Templeton y Copello, 2005).

Respecto a la relación entre el consumo de sustancias y la conducción de vehículos, obtuvimos que los jóvenes que han consumido cocaína o éxtasis es más probable que hayan conducido bajo los efectos del alcohol en el último año, que hayan ido con alguien que conducía bajo los efectos de esta sustancia y que hayan conducido bajo los efectos de otras drogas como el cannabis o la cocaína alguna vez en su vida. Respecto a los problemas derivados de conducir bajo el efecto de sustancias, también es más probable que estos jóvenes hayan sido multados, detenidos o que hayan sufrido algún accidente de tráfico. En el estudio de Calafat et al. (2000) se recoge que el 38\% de los jóvenes utiliza su coche para desplazarse cuando salen de marcha, el 51.5\% utiliza el de sus amigos y un alto porcentaje ha conducido bajo los efectos del alcohol (50.7\%) o de otras drogas (36.8\%). La consecuencia más grave de esta conducta son los accidentes de tráfico que con frecuencia se saldan con víctimas mortales (Farke y Anderson, 2007; Greemblatt, 2000). Por lo tanto, el grupo de consumidores de psicoestimulantes tienen un mayor riesgo de sufrir accidentes de tráfico $u$ otros problemas relacionados con la conducción aunque Infante, Barrio y Martín (2003) señalan que no hay una relación específica entre psicoestimulantes y accidentes de tráfico, ya que en la mayor parte de los accidentes detectaron que hay un policonsumo de sustancias y el alcohol tiene un lugar destacado. Calafat et al. (2007) apuntan que haber conducido vehículos bajo los efectos del alcohol o estar dispuesto a ir en un vehículo que conduce alguien que haya bebido son dos factores de riesgo que explican el consumo de drogas. Por ello, es necesario diseñar intervenciones para reducir este tipo de conductas de riesgo que con frecuencia tienen fatales consecuencias y que no estén dirigidas únicamente hacia el consumo de alcohol sino también hacia el de otras drogas que conocemos que también interfieren en la capacidad de conducción de vehículos (Blows, Ivers, Connors, Ameratunga, Wodward y Norton, 2005). 
Analizamos la relación entre consumo de drogas y relaciones sexuales debido a la frecuencia de esta asociación. Los jóvenes consideran que estas sustancias facilitan el inicio de relaciones sexuales y la realización de prácticas sexuales poco frecuentes, siendo el alcohol y la cocaína las mejor valoradas para ello (Calafat et al., 2008; Sumnall et al., 2007). En el presente estudio hemos obtenido que es más probable que los jóvenes que han consumido cocaina o éxtasis hayan mantenido relaciones sexuales completas con menor edad y que en el último año las hayan mantenido con un mayor número de personas. Estos datos coinciden con otros estudios (Bellis et al., 2008; Bellis et al., 2000; Hughes et al., 2009; Castilla, Barrio, Belza y de la Fuente, 1999) que señalan que el consumo de sustancias como el alcohol, cannabis, cocaína o éxtasis antes de los 16 años está relacionado con un inicio más temprano en las relaciones sexuales. Además, los consumidores de drogas en el ámbito de salir de marcha han mantenido relaciones sexuales con un mayor número de parejas e incluso es más probable que hayan pagado por mantener relaciones sexuales.

Como consecuencia de la asociación entre consumo de drogas y relaciones sexuales encontramos, al igual que otros estudios (Becoña, Juan, Calafat y Ros, 2008; Calafat et al., 2008, 2010, Bellis et al., 2000; Bellis et al., 2008; Castilla et al., 1999; Coleman y Cater, 2005; Donovan y McEwan, 1995; Rodríguez et al., 2007), que es más probable que los consumidores de cocaína o éxtasis hayan mantenido relaciones sexuales sin utilizar preservativo o alguna otra forma de control del embarazo y que lo hayan hecho bajo los efectos del alcohol o de otras drogas. Esto tiene como consecuencia los embarazos no deseados y el contagio de enfermedades de transmisión sexual.

Pero ¿qué es lo que explica que se realicen estas conductas de riesgo bajo los efectos de drogas? Para Bellis et al. (2008) que los jóvenes realicen estas conductas sexuales de riesgo se debe a la alteración que produce el consumo de drogas en la toma de decisiones incrementando la probabilidad de que mantengan relaciones sexuales sin protección. En esta línea, Leeman, Grant y Potenza (2009) apuntan que hay una clara relación entre consumo de sustancias y desinhibición, que la intoxicación por sustancias produce en el cerebro alteraciones similares al deterioro cognitivo que se encuentra en sujetos con abuso de sustancias crónico e incluso a la desinhibición característica de las lesiones en el lóbulo frontal. Gonzalez et al. (2005) señalan que el principal factor que explica la realización de prácticas sexuales de riesgo en consumidores de drogas como la cocaína o la heroína es el rasgo de personalidad de búsqueda de sensaciones, mientras que Espada, Antón y Torregrosa (2008) señalan el autoconcepto como factor predictor de la realización de conductas de riesgo bajo los efectos de las drogas (sin el alcohol) por encima de la búsqueda de sensaciones. Por otro lado, Gullette y Lyons (2006) señalan que la baja autoestima es el factor que explica que algunos jóvenes realicen consumos más altos de alcohol, mantengan relaciones sexuales con un mayor número de parejas y tengan conductas de riesgo como la no utilización de preservativos. Además, la presencia del trastorno de personalidad antisocial, frecuente en consumidores de cocaína (López y Becoña, 2006b, c), también aumenta la probabilidad de que la persona lleve a cabo conductas sexuales de riesgo, ya que este trastorno se caracteriza por la impulsividad y la irresponsabilidad (Compton, Cottler, Shillington y Price, 1995).

Por lo tanto, y en función de los resultados del presente estudio, no podemos olvidar la importancia que debe tener en esta población una adecuada intervención dirigida a evitar que realicen conductas sexuales de riesgo (Bellis et al., 2000; Gullette y Lyons, 2006), tanto para evitar los embarazos no deseados como enfermedades de transmisión sexual como puede ser el VIH. En el estudio publicado por el Registro Nacional de casos de SIDA (2008) se recoge que el 30\% de los nuevos casos de SIDA diagnosticados en el año 2007 son consecuencia de mantener relaciones sexuales sin protección.

Por último, realizamos un análisis de regresión logística para conocer qué variables de las analizadas en el presente estudio explicaban el consumo de psicoestimulantes. Encontramos que dicho consumo está relacionado con una mayor frecuencia de borracheras en el último año y con conductas de riesgo como ir en un coche cuyo conductor había bebido, conducir bajo los efectos de sustancias psicoactivas, una edad de inicio más temprana en las relaciones sexuales completas, no utilizar ningún método anticonceptivo y mantener relaciones sexuales bajo los efectos de alguna droga.

Las variables que han sido analizadas se centran en aspectos relacionados con salir de marcha que son el reflejo de la importancia de algunos de los cambios que se están viviendo en nuestra sociedad en los últimos años y que son claves para explicar los nuevos patrones de consumo de drogas y de problemas derivados de dicho consumo entre los más jóvenes (embarazos no deseados, accidentes de tráfico, enfermedades de transmisión sexual, etc.). A todo esto, Calafat et al. (2004) lo han denominado como Modelo de Diversión Hegemónico, caracterizado por importantes consumos de alcohol y drogas y por mantener relaciones sexuales con parejas diferentes (Bellis et al., 2000). Esta forma de diversión nocturna surge en las zonas turísticas y rápidamente se ha extendido a otros puntos de nuestro país. A pesar de ser un fenómeno reciente no podemos olvidar su relevancia ya que estamos padeciendo en la actualidad algunas de las consecuencias negativas derivadas del mismo (accidentes de tráfico, embarazos no deseados o el incremento de las enfermedades de transmisión sexual). De aquí, la necesidad de diseñar intervenciones preventivas especificas para este ámbito debido a las importantes consecuencias negativas que se están produciendo (Calafat, Juan y Duch, 2009).

Entre las limitaciones del presente estudio, debemos señalar que se trata de un estudio transversal y es necesario llevar a cabo estudios longitudinales que analicen la evolución de los jóvenes que participan activamente en el fenómeno de salir de marcha y las posibles consecuencias que en el futuro puedan tener los importantes consumos de sustancias psicoactivas que vienen realizando. También que la información es autoinformada y no se ha hecho una verificación biológica de los consumos de drogas. Y, finalmente, que se podría haber analizado un mayor número de variables en el 
estudio, aunque ello es difícil por el tipo de muestra utilizada y por el procedimiento de entrevista realizado.

\section{AGRADECIMIENTOS}

Este estudio se ha realizado gracias al Convenio de Colaboración entre la Consellería de Sanidade-Servizo Galego de Saúde y la Universidad de Santiago de Compostela para el apoyo de las acciones de formación e investigación en la Subdirección General de Gestión Sosicosanitaria y Salud Mental, Dirección de Asistencia Sanitaria. Servizo Galego de Saúde, Santiago de Compostela.

\section{REFERENCIAS}

Ballester, R. y Gil, M. D. (2009). ¿Por qué los jóvenes se dan atracones de alcohol los fines de semana? Revista de Psicopatología y Psicología Clínica, 14, 25-35.

Becoña, E., Juan, M., Calafat, A. y Ros, M. (2008). Razones para no aceptar una relación sexual en jóvenes que se divierten en contextos recreativos nocturnos en función del género y de la embriaguez. Adicciones, 20, 357-364.

Bellis, M. A., Hale, G., Bennet, L. A., Chaves, M. y Kilfoyle, M. (2000). Ibiza uncovered: Changes in substance use and sexual behaviour among young people visiting an international night-life resort. International Journal of Drug Policy, 11, 235-244.

Bellis, M. A., Hughes, K., Bennett, A. y Thomson, R. (2003). The role of an international nightlife resort in the proliferation of recreational drugs. Addiction, 98, 1713-1721.

Bellis, M. A., Hughes, K., Calafat, A., Juan, M., Ramon, A., Rodriguez, J. A., Phillips-Howard, P. (2008). Sexual uses of alcohol and drugs and the associated health risks: A cross sectional study of young people in nine European cities. BMC Public Health, 8, 1-11.

Blows, S., Ivers, R.Q., Connors, J., Ameratunga, S., Wodward, M. y Norton, R. (2005). Marijuana use and car crash injury. Addiction, 100,605-611.

Cadaveira, F. (2009). Alcohol y cerebro adolescente. Adicciones, 21, 9-14.

Calafat, A., Fernandez, C., Juan, M., Anttila, A., Bellis, M., Bohrn, K.,... Zavatti, P. (2004). Cultural mediators in hegemonic nightlife. Palma de Mallorca: Irefrea.

Calafat, A., Fernández, C., Juan, M. y Becoña, E. (2005). Gestión de la vida recreativa: ¿Un factor de riesgo determinante en el uso reciente de drogas? Adicciones, 17, 337-347.

Calafat, A., Fernández, C., Juan, M. y Becoña, E. (2007). Vida recreativa nocturna de los jóvenes españoles como factor de riesgo frente a otros más tradicionales. Adicciones, 19, 125-132.

Calafat, A., Juan, M., Becoña, E., Castillo, A., Fernández, C., Franco, M., Ros, M. (2005). El consumo de alcohol en la lógica del botellón. Adicciones, 17, 193-202.
Calafat, A., Juan, M., Becoña, E., Fernández, C., Gil, E., Palmer, A.,... Torres, M. A. (2000). Salir de marcha y consumo de drogas. Madrid: Plan Nacional sobre Drogas.

Calafat, A., Juan, M., Becoña E. y Mantecón, A. (2008). Qué drogas se prefieren para las relaciones sexuales en los contextos recreativos. Adicciones, 20, 37-48.

Calafat, A., Juan, M., y Duch, M. A. (2009). Intervenciones preventivas en contextos recreativos nocturnos: revisión. Adicciones, 21, 387-414.

Calafat, A., Juan, M., Becoña, E., Mantecón, A. y Ramón, A. (2009). Sexualidad de riesgo y consumo de drogas en el contexto recreativo. Una perspectiva de género. Psicothema, 21, 227-233.

Castilla, J., Barrio, G., Belza, M. J. y de la Fuente, L. (1999). Drug and alcohol consumption and sexual risk behaviour among young adults: Results from a national survey. Drug and Alcohol Dependence, 56, 47-53.

Coleman, L. y Cater, S. (2005). Underage "risky" drinking: Motivations and outcomes. York: Joseph Rowntree Foundation.

Compton, W. M., Cottler, L. B., Shillington, A. M. y Price, R. K. (1995). Is antisocial personality disorder associated with increased HIV risk behaviors in cocaine users? Drug and Alcohol Dependence, 37, 37-43.

Cortés, M. T., Espejo, B. y Giménez, J. A. (2008). Aspectos cognitivos relacionados con la práctica del botellón. Psicothema, 20, 396402.

Cortés, M. T., Espejo, B., Martín, B. y Gómez, C. (2010). Tipologías de consumidores de alcohol dentro de la práctica del botellón en tres ciudades españolas. Psicothema, 22, 363-368.

Donovan, C. y McEwan, R. (1995). A review of the literature examining the relationship between alcohol use and HIV-related sexual risk-taking in young people. Addiction, 90, 319-328.

Espada, J. P., Antón, F. A. y Torregrosa, M. A. (2008). Autoconcepto y búsqueda de sensaciones como predictores de las conductas sexuales bajo los efectos de las drogas en universitarios. Salud y Drogas, 8, 137-155.

Farke, W. y Anderson, P. (2007). El consumo concentrado de alcohol en Europa. Adicciones, 19, 333-340.

Gonzalez, R., Vassileva, J., Bechara, A., Grbesic, S., Sworowski, L., Novak, R. M.,...Martin, E. M. (2005). The influence of executive functions, sensation seeking, and HIV serostatus on the risky sexual practices of substance-dependent individuals. Journal of International Neuropsychological Society, 11, 121-131.

Greenblatt, J. C. (2000). Patterns of alcohol use among adolescents and associations with emotional and behavioural problems. Rockville, MD: OAS Working Paper.

Gullette, D. L. y Lyons, M. A. (2006). Sensation seeking, self-esteem, and unprotected sex in college students. Journal of the Association of Nurses in AIDS Care, 17, 23-31.

Hingson, R. P., Edwards, E. M., Heeren, T. y Rosenbloom, D. (2009). Age of drinking onset and injuries, motor vehicle, and physical fights after drinking and when not drinking. Alcoholism: Clinical and Experimental Research, 33, 783-790. 
Hughes, K, Bellis, M. A., Whelan, G., Calafat, A., Juan M. y Blay, N. (2009). Alcohol, drogas, sexo y violencia: riesgos y consecuencias para la salud entre los jóvenes turistas británicos en las islas Baleares. Adicciones, 21, 265-278.

Infante, C., Barrio, G. y Martín, E. (2003). Tendencias, caracteristicas y problemas asociados al consumo de drogas recreativas en España. Adicciones, 15 (Supl. 2), 77-96.

Leeman, R. F., Grant, J. E. y Potenza, M. N. (2009). Behavioral and neurological foundations for the moral and legal implications of intoxication, addictive behaviors and disinhibition. Behavioral Science and the Law, 27, 237-259.

López, A. y Becoña, E. (2006a). El consumo de alcohol en las personas con dependencia de la cocaina que están en tratamiento. Psicología Conductual, 14, 235-245.

López, A. y Becoña, E. (2006b). Consumo de cocaina y psicopatología asociada: una revisión. Adicciones, 18, 161-196.

López, A. y Becoña, E. (2006c). Patrones y trastornos de personalidad en personas con dependencia de la cocaina en tratamiento. Psicothema, 18, 577-582.

Miller, J. W., Naimi, T. S., Brewer, R. D. y Jones, S. E. (2007). Binge drinking and associated health risk behaviours among high school students. Pediatrics, 119, 76-85.

Observatorio Español sobre Drogas (2009). Encuesta Estatal sobre Uso de Drogas en Estudiantes de Enseñanzas Secundarias (ESTUDES). Recogido el 10 de mayo de 2010 en http://www.pnsd.msc.es/ Categoria2/observa/pdf/Estudes2008.pdf.

Registro Nacional de Casos de Sida (2008). Vigilancia epidemiológica del VIH-Sida en España. Madrid: Instituto de Salud Carlos
III, Centro Nacional de Epidemiología. Informe semestral, n 1, 2008.

Rodríguez, A., Hernán, M., Cabrera, A., García, J. M. y Romo, N. (2007). ¿Qué opinan adolescentes y jóvenes sobre el consumo de drogas recreativas y conductas sexuales de riesgo? Adicciones, 19, 153-167.

Rosnow, R. L. y Rosenthal, R. (1996). Beginning behavioural research. A conceptual primer. Upper Saddle River, N. J.: Practice-Hall, Inc. Schnitzer, S., Bellis, M. A., Anderson, Z., Hughes, K., Calafat, A., Juan, M. y Kokkevi, A. (2010). Nightlife violence: A genderspecific view on risk factors for violence in night-life settings. A cross-sectional study in nine European countries. Journal of Interpersonal Violence, 25, 1094-112.

Siliquini, R., Morra, A., Versino, E. y Renga, G. (2005). Recreational drug consumers: Who seeks treatment? European Journal of Public Health, 15, 580-586

Steinberg, L. (2010). A behavioral scientist looks at the science of adolescent brain development. Brain and Cognition, 72, 160-164.

Sumnall, H. R., Beynon, C. M., Conchie, S. M., Riley, S. C. y Cole, J. C. (2007). An investigation of the subjective experiences of sex after alcohol or drug intoxication. Journal of Psychopharmacology, 21, 527-537.

Sussman, S., Skara, S. y Ames, S. L. (2008). Substance abuse among adolescents. Substance Use \& Misuse, 43, 1802-1828.

Velleman, R. D. B., Templeton, L. J. y Copello, A. G. (2005). The role of the family in preventing and intervening with substance use and misuse: A comprehensive review of family interventions, with a focus on young people. Drug and Alcohol Review, 24, 93-109. 